\title{
Cognition and return to work after mild/moderate traumatic brain injury: A systematic review
}

\author{
Karthik Mani ${ }^{\mathrm{a}, *}$, Bryan Cater $^{\mathrm{b}}$ and Akshay Hudlikar ${ }^{\mathrm{c}}$ \\ ${ }^{a}$ Assistant Director, External and Regulatory Affairs, NBCOT, USA \\ b Occupational Therapist, Fit for Work, St. John's, NF, Canada \\ ${ }^{\mathrm{c} C l i n i c a l}$ Specialist, Aegis Therapies, USA
}

Received 15 June 2016

Accepted 18 October 2016

\begin{abstract}
.
BACKGROUND: Approximately two percent of the United States population are traumatic brain injury (TBI) survivors. The unemployment rate among them is substantial. Cognitive skills are essential to perform any job.

OBJECTIVE: We analyzed the literature on cognitive rehabilitation (CR) related to mild/moderate TBI to learn the influence of cognition on return to work (RTW) post TBI.

METHODS: We conducted a systematic review of the studies on CR related to RTW post TBI that were published between 2000 and 2015.

RESULTS: We critically reviewed 30 studies that met the inclusion criteria. Ten studies highlighted cognition as a predictor variable, seven studies demonstrated support for cognitive testing in RTW assessments, and 13 studies showed the efficacy of CR in facilitating RTW post TBI.

CONCLUSION: Cognition plays a significant role in predicting and facilitating RTW in patients with TBI.
\end{abstract}

Keywords: Cognitive rehabilitation, vocational outcome, evidence-based review

\section{Introduction}

Over the past decade, the morbidity and mortality rates for Traumatic Brain Injury (TBI) have increased. The Centers for Disease Control and Prevention (CDC) estimated that the combined rates of TBI related emergency department visits, hospitalizations, and deaths were 823.7 per 100,000 in 2010 [1]. Approximately two percent of the United States

*Address for correspondence: Karthik Mani, $339 \mathrm{~W}$. Side Drive, Gaithersburg, MD 20878, USA. Tel: +1 301250 6864; E-mail: rkhitak04@gmail.com.
(US) population live with disabilities secondary to brain injuries [2]. Focused rehabilitation efforts are imperative to ensure the quality of life (QOL) of TBI survivors.

The ultimate aim of any rehabilitation program is to achieve the highest possible level of function and QOL. Among the myriad factors that affect QOL, Return to Work (RTW) is considered a critical one [3], as work plays a valuable role in a person's life [4] and employment is considered a positive factor that influences QOL [5]. Using the World Health Organization's model of disability, Pierce and Hanks [6] cited participation as a strong predictor of life 
satisfaction among individuals with disability. Return to work enhances self-esteem and allows a person with disability to return to being a productive member of the society.

Traumatic brain injuries range in severity from mild to severe and are typically classified based on the duration of unconsciousness, extent of posttraumatic amnesia, and findings on structural imaging [7]. Traumatic brain injury and other neurological dysfunctions prevent RTW by affecting physical, psychosocial, cognitive, and visual-perceptual skills [5, $8,9]$. Cognitively, they impair effective job performance by creating difficulties in learning new job tasks and attending to tasks [10]. Regardless of the severity level, TBI affects an individual's ability to work. Boeing et al. stated that even a mild TBI can affect one's ability to work "as a result of changes in interpersonal relationships, short term memory, executive skills, and behavior" [11, p.35]. Coetzar et al. [12] reported that individuals with moderate to severe TBI showed a significant change in their work status post-injury when compared to that of pre-injury. Among TBI survivors, the unemployment rate was reported to be substantially high $[13,14]$.

Cognitive skills are most affected in brain injuries, to an extent that is proportional to the severity of the injury [15]. Mahar and Fraser stated that "disruption of cognitive functioning is irrefutably the primary deficit following most forms of brain injury" [9, p. 54]. The cognitive skills that are affected due to TBI include attention, memory, processing speed, problem solving, judgment, executive functioning, self-awareness, numeracy and literacy ability, multitasking ability, and verbal learning and comprehension [9, 16-18]. A variety of cognitive intervention methods comprised of both restoring and compensating interventions were used to treat cognitive deficits in TBI [19]. Cognitive recovery post TBI was reported to be less predictable and influenced by several factors [20].

Multiple factors influence RTW outcomes in TBI rehabilitation. Pre-injury demographics (e.g., age, gender, education, race, marital status, pre-injury productivity, type of pre-injury occupation), injuryrelated variables (e.g., severity, mechanism of injury, duration of coma, length of post-traumatic amnesia, pattern of recovery, length of hospital stay, level of disability at the time of admission to rehabilitation), and post-injury variables (e.g., comorbid psychiatric diagnoses, duration since injury, physical rehabilitation services received, job-placement services received, psychosocial services received, disability rating outcomes, functional status at discharge) influence RTW outcome post TBI [19-27]. Public awareness on TBI and recovery [9], and availability of support services for individuals with TBI were also found to influence RTW post TBI [28].

Since there is a complex interplay between cognition, TBI, and RTW, to deliver effective vocational rehabilitation and facilitate community integration, it is critical for rehabilitation professionals to understand the relationship between "cognition" and "RTW post TBI." This systematic review synthesizes evidence to examine the relationship between cognition and RTW among TBI survivors with mild or moderate TBI. The underlying objective is to identify whether cognition based interventions facilitate RTW post TBI.

\section{Methodology}

For this systematic review, the authors adopted an approach from a guidebook on critical appraisal for evidence-based practice [29]. This approach advises the researchers completing a systematic review to formulate a focused clinical question, determine inclusion and search strategies, critically evaluate the studies, and summarize the findings. The PIO (Population, Intervention, \& Outcome) question was formulated based on the consensus among the authors' on the clinical utility value of the question. This review answers the following PIO question: What is the evidence on the relationship between cognition (intervention) and RTW (outcome) post TBI (population)?

\subsection{Inclusion and exclusion criteria}

To synthesize the latest evidence, only articles published between 2000 and 2015 were selected for review. Only articles published in English were chosen as English is considered a universal language. In addition, articles were selected only if the study samples were adults between 18 and 65 years of age. This age range was applied because work is considered an adult occupation and vocational services were typically provided, in practice, to individuals of working age [30]. Studies related to acquired brain injury (ABI) were also selected for review if the study's sample included individuals with TBI. Acquired brain injury studies were part of the inclusion criteria since ABI includes all types of TBI [31]. 
Table 1

Databases and journals searched for this review

\begin{tabular}{l}
\hline Databases \\
- Cumulative Index to Nursing and Allied Health Literature \\
- Google Scholar \\
- OT CATS \\
- OT Seeker \\
- Proquest \\
- Pubmed \\
- EBSCOHost \\
Journals \\
- Neurorehabilitation Journals \\
- Occupational Rehabilitation Journals \\
- Occupational Therapy Journals \\
- Brain Injury Journals \\
- Rehabilitation Medicine Journals \\
- Physical Medicine and Rehabilitation Journals \\
- Community Rehabilitation Journals \\
- Disability and Rehabilitation Journals \\
- Lancet Neurology
\end{tabular}

Articles were excluded if the study samples included individuals: (a) with a diagnosis of severe TBI, (b) with a diagnosis other than TBI, or (c) who did not meet the 18 to 65 age range. However, the authors did consider one study with a large sample size [26] in which the sample included individuals with severe TBI, due to its strong relevance to the PIO question and because $64 \%$ of the sample were individuals with mild and moderate TBI. Articles were also excluded if the focus was not on cognitive skills or cognitive rehabilitation. In addition, gray literature such as dissertations, reports, and conference abstracts were excluded.

\subsection{Search strategy}

The authors used a wide range of sources (Table 1) to identify articles for this review. The authors selected these sources because of their relevance to the PIO question under investigation. In order to retrieve the most relevant results from the databases and journals, all search entries included the term TBI, along with a combination of the following keywords: "cognition," "return to work," "work resettlement," "vocational rehabilitation," "community integration," "cognitive rehabilitation," "cognitive intervention," "adults," "mild traumatic brain injury," and "moderate traumatic brain injury."

The authors independently searched the databases and journals, dividing the sources among themselves based on their preference and ability to access full-text articles. The abstracts that emerged were carefully reviewed by applying the inclusionexclusion criteria.
To classify the obtained evidence, the authors used the levels of evidence recommended by the American Occupational Therapy Association, Inc., in the document "Guidelines for Systematic Reviews" [32]. The guidelines define five levels of evidence. Level I includes systematic reviews, meta-analyses, and randomized controlled trials (RCT's); level II includes two groups, nonrandomized studies; level III includes one group, nonrandomized studies; level IV includes descriptive studies that include analysis of outcomes; and level V includes case reports and expert opinion with narrative literature and consensus statements.

\section{Results}

The search of the databases and journals yielded a total of 258 articles. Of the 258 articles, 224 articles did not meet the inclusion criteria after the abstracts were reviewed. Of the remaining 34 articles, four were further excluded after the full text was analyzed because they examined non-cognitive variables, job search and placement services, vocational services, and case coordination services related to RTW post TBI. Therefore, the literature search yielded a total of 30 applicable articles with varying levels of evidence. Table 2 presents the evidence summary. Given the heterogeneity of the obtained evidence, the authors examined the articles for similarities in findings and grouped them into three themes. Of the 30 articles, 10 articles identified cognition as an important predictor variable of RTW post TBI, seven articles identified cognition as a critical variable in RTW assessments post TBI, and 13 articles supported the use of cognitive rehabilitation to facilitate RTW post TBI. Key findings of the articles reviewed are summarized below.

\subsection{Cognitive variables predict return to work post traumatic brain injury}

Ten studies identified either single or multiple cognitive variables as predictors of RTW post TBI, including 4 Level I studies [22, 25, 33, 34], one Level II study [23], 2 Level III studies [21, 26], and 3 Level IV studies [24, 35, 36]. Executive functioning emerged as the most commonly cited predictor variable of RTW post TBI and was cited in three studies $[23,34,35]$. The Disability Rating Scale (DRS) outcome, which rates the cognitive ability to perform self-care activities, was identified as a predictor 


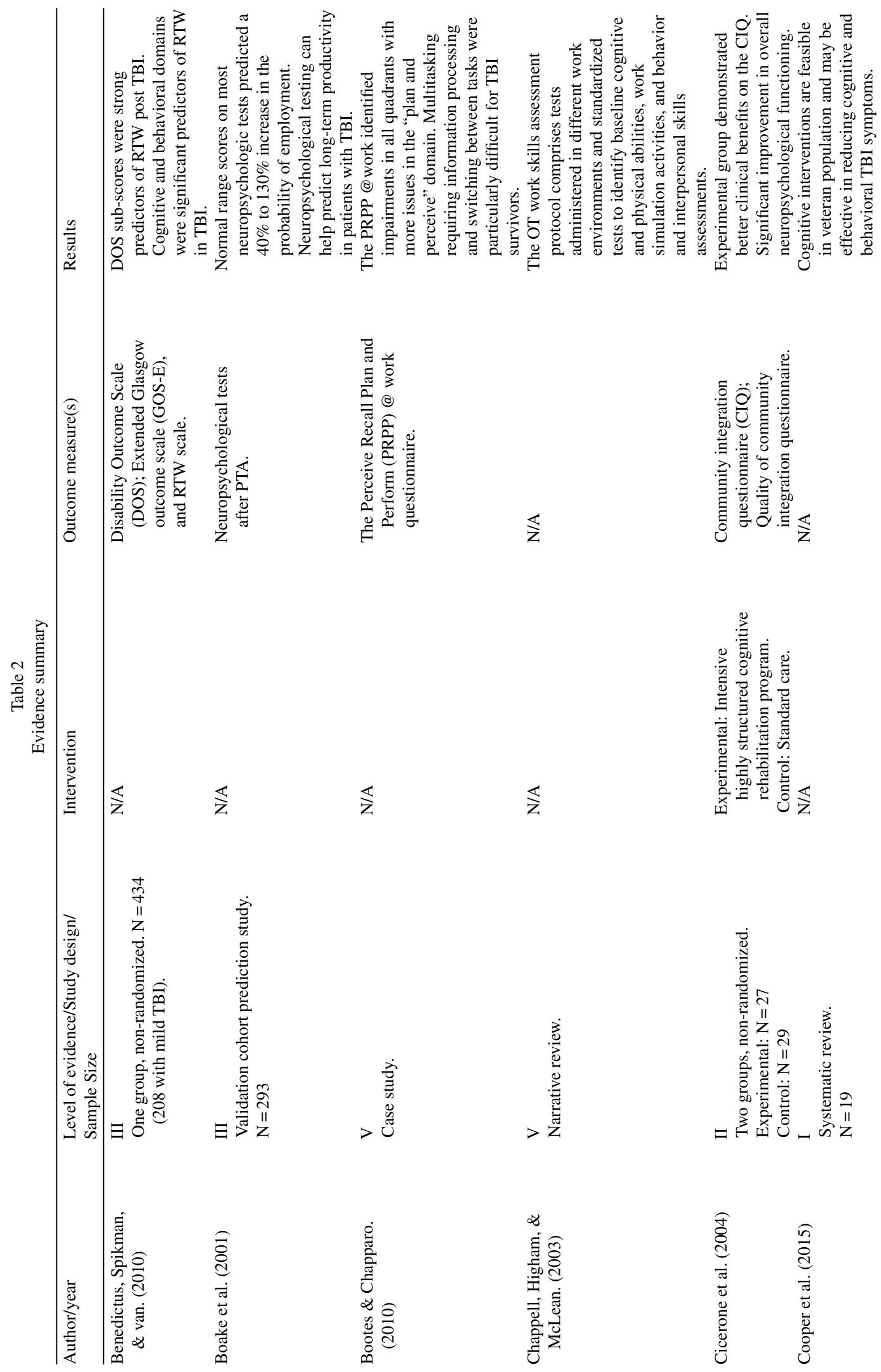




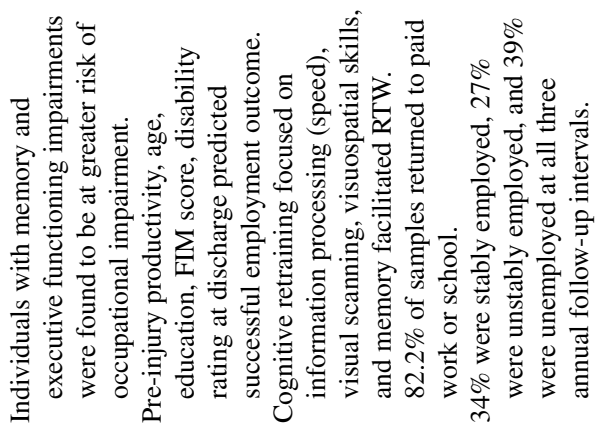
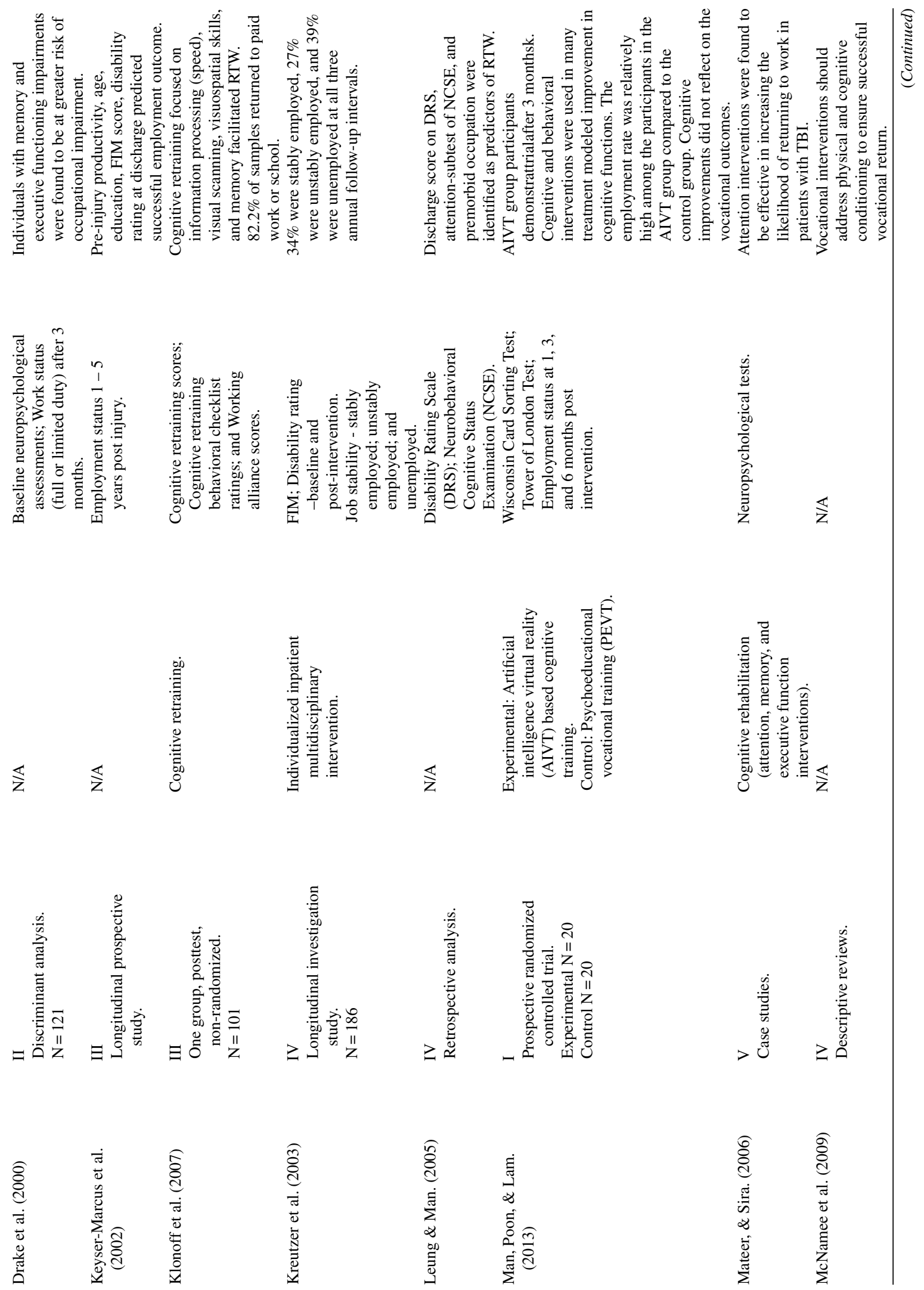

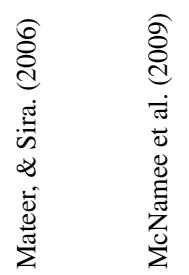




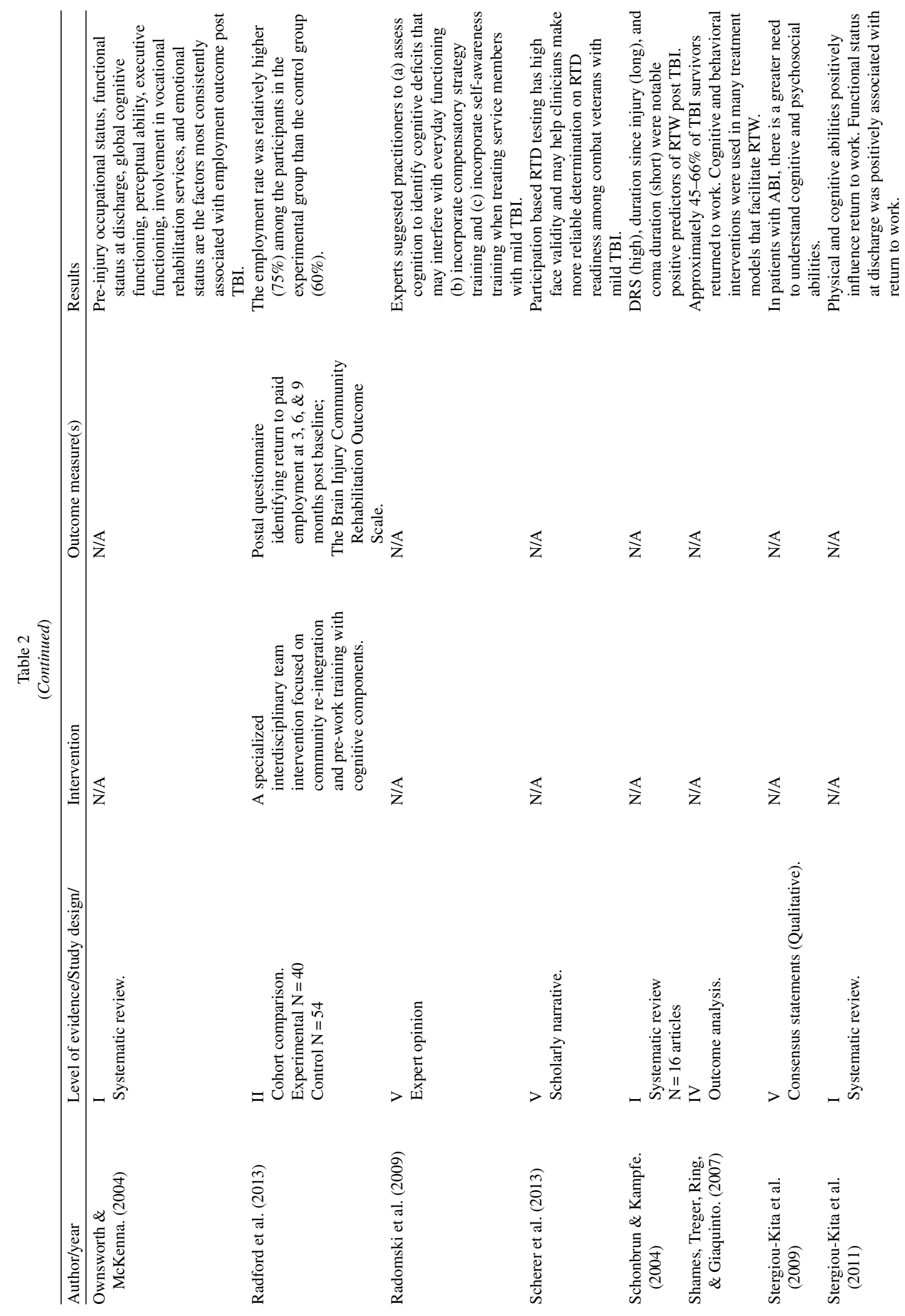



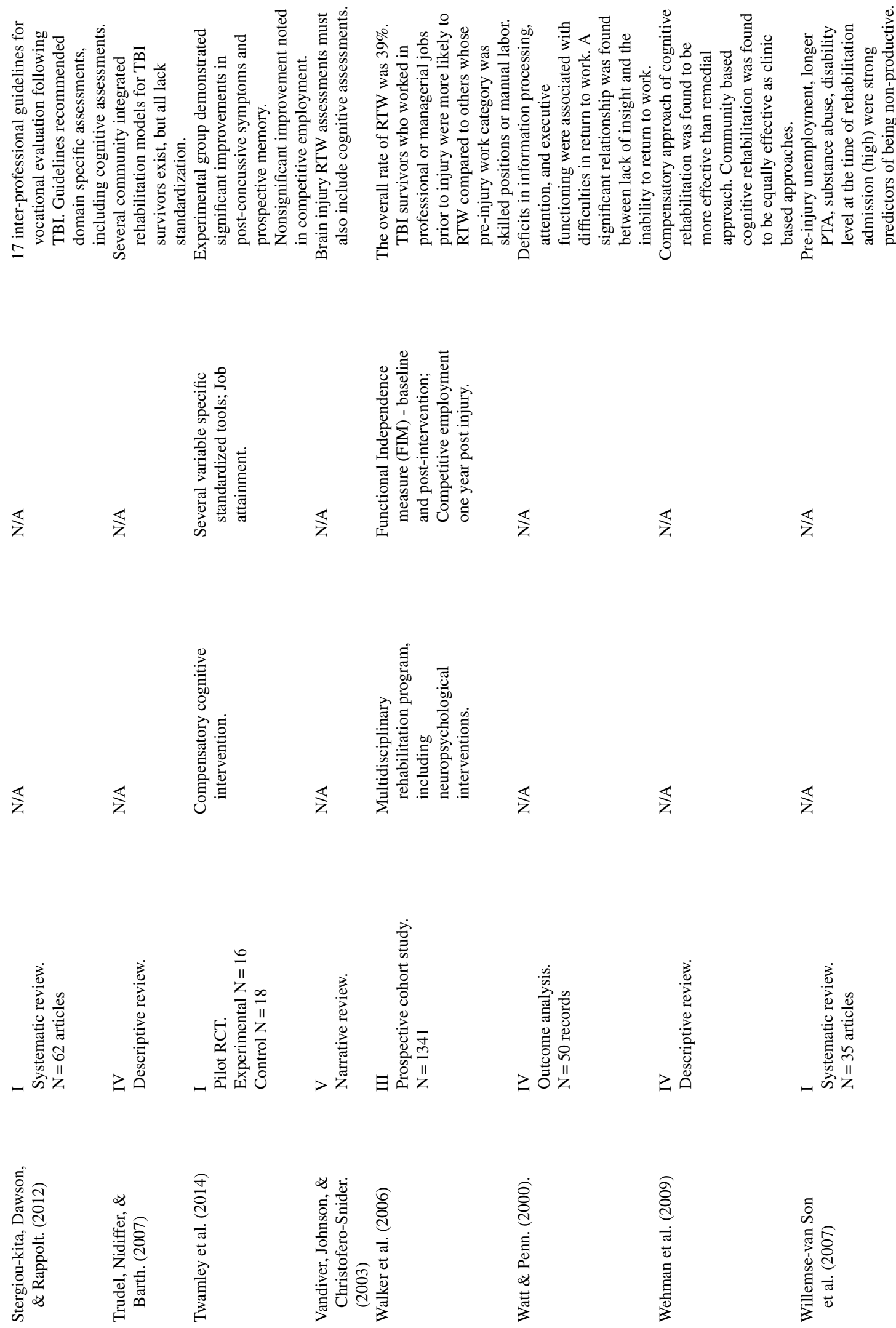

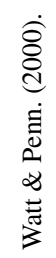

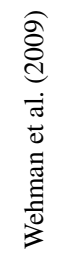

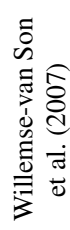

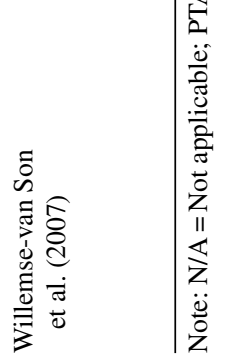


variable in two studies [24, 37]. Attention [24, 35], memory [23, 33], and verbal skills [22, 23] were also cited in two studies each as predictors of RTW post-TBI. Other cognitive factors cited in the studies included: performance IQ [22]; the Functional Independence Measure (FIM ${ }^{\mathrm{TM}}$ ) outcome, which also assesses cognitive disability [21]; global cognitive functioning [34]; perceptual ability [34]; and processing speed [35].

\subsection{Cognitive assessment as an inherent component in return to work assessments}

Seven studies identified cognition as a critical variable in RTW assessments of patients with mild/moderate TBI. One Level I systematic review suggested cognitive testing under its proposed domain-specific clinical guidelines for vocational evaluation post TBI. Three other Level V articles (38, 39,40 ) highlighted the importance of cognitive testing. Cognitive testing was a part of RTW assessments, a participation based and a process oriented criterion referenced, described by 2 level V studies [37,41]. All of these studies recommended standardized testing of baseline cognitive abilities during work skills assessments. Further, a Level III validation cohort study [10] highlighted the predictive ability of neuropsychological tests in predicting RTW and long-term productivity among patients with TBI.

\subsection{Cognitive rehabilitation facilitate return to work post traumatic brain injury}

Thirteen studies in this review demonstrated the efficacy of cognitive interventions in facilitating RTW post TBI. Six of the studies were literature reviews, five studies were efficacy studies with one or more comparison groups, one was a single group study, and one was an article with expert opinions.

Several review studies identified cognitive intervention as a viable intervention to successfully facilitate RTW in patients with TBI. One Level I systematic review [19] highlighted the role of cognitive interventions in improving neurobehavioral symptoms and successful RTW. Four Level IV descriptive reviews [8, 42-44] recommended cognitive interventions, with increased emphasis on compensatory strategies, to facilitate RTW.

Evidence from 2 Level I studies [45, 46] and 2 Level II studies [47, 48] reported on the benefit of cognitive interventions to accelerate RTW. In these studies, cognitive interventions were focused on improving memory, post-concussive symptoms, and neuropsychological functioning.

Two studies identified the relationship between retraining specific cognitive skills and RTW post TBI. Klonoff et al. [49] identified that cognitive retraining associated with processing speed, visual perceptual skills, and memory enhanced successful RTW. Through case studies, Mateer and Sira [50] identified that attention interventions would increase the likelihood of RTW. Further, they recommended individually tailored, context-based cognitive interventions focused on attention, memory, and executive functioning.

Expert opinions favor cognitive rehabilitation in facilitating RTW post TBI. In their article on clinical guidelines for treating service members with mild TBI, Radomski et al. [51] recommended that OT practitioners assess and treat cognitive deficits that interfere with everyday functioning. Radomski et al. also recommended that practitioners incorporate compensatory strategies and self-awareness training as part of the OT intervention.

One study reported on the influence that pre-injury occupational factors have on RTW post TBI. Walker et al. [52] examined the relationship between preinjury occupational category and RTW post TBI and concluded that after a multidisciplinary intervention with neuropsychological treatment, individuals who worked in a professional/managerial role prior to injury were more successful in RTW than individuals who were skilled/manual laborers.

\section{Discussion}

The findings of this review show that cognition plays an important role in RTW post TBI. This review systematically assesses published studies dealing with cognition, mild/moderate TBI, and RTW to examine how cognition influences RTW post TBI. This study adds to the existing research on cognitive rehabilitation in TBI by emphasizing the importance of cognitive assessment and intervention in facilitating RTW. Caution must be exerted when generalizing the findings as the studies included in this review varied in design (intervention studies, prospective cohort studies, retrospective analysis, case series, and descriptive articles with expert opinions) and were conducted in different settings with different demographic and clinical characteristics. However, despite the differences, the authors believe that this review highlights the critical role that 
cognition plays in facilitating and sustaining RTW post TBI.

This review identifies cognition as one of the significant predictor variables of RTW post TBI. It is not surprising that executive function emerged as a significant predictor since it is considered an essential skill to perform any kind of work. Matheson, Dodson, and Wolf stated that "executive functions control emotional responses and provide coordination and integration of the basic cognitive processes" [53], which are much needed skills for effective job performance. Matheson et al. added that the demand for executive functioning on a job was positively associated with job complexity and negatively associated with job organization. Other cognitive skills (attention, memory, information processing, and verbal skills) that surfaced in this review as predictive cognitive skills were identified in the RTW literature as essential cognitive skills for effective job performance $[42,54,55]$. It is interesting to note that these skills were also reported to be significantly affected in patients with mild TBI [56, 57].

Cognitive evaluations, often in the form of neuropsychological evaluations, were reported to be effective in identifying an individual's capacity to work [11] because they provided valuable information on the assessed individual's strengths and deficits. Such evaluations can help rehabilitation professionals understand TBI survivors and plan effective treatment for them. Although several studies in this review supported the use of cognitive assessment as part of RTW assessments, the method of testing varied widely across the studies. Some studies used standardized tests $[10,38]$ while other studies used task and domain specific assessments [37, 58]. Interestingly, one study interviewed the employer to gain information about the cognitive capacity of an employee with TBI [41]. This variability creates an inconsistency in testing cognitive abilities in RTW assessments. Wasiak et al. [59] argued that the inconsistency and lack of comprehensiveness of RTW measurements are factors that limit the understanding of RTW. Hoofien et al. [60] also highlighted the inconsistency of the outcome measures used in TBI rehabilitation. On the other hand, Bayley et al. [61] advocated for tailored cognitive assessments based on patients' premorbid cognitive characteristics and life goals. The findings reveal a lack of a comprehensive RTW cognitive assessment tool focusing TBI survivors.

Cognitive rehabilitation for individuals with TBI was reported to be an effective intervention. The lit- erature reviews by Cicerone et al. revealed that the treatments for attention, memory, functional communication, and executive functioning after TBI were effective [48, 62]. The current findings add to this literature.

Two notable observations emerged during the analysis of the literature. First, cognitive rehabilitation post TBI focused more on using compensatory strategies than remedial strategies. Several studies in this review recommended the use of compensatory strategies [19, 42, 43, 49]. Cernich et al. [63] stated that training in the use of supportive devices remains central to independent functioning of individuals with TBI in the community.

Second, both the review and intervention studies highlighted multidisciplinary/interdisciplinary rehabilitation practices in facilitating RTW post TBI. This is consistent with some previous findings, which favored multidisciplinary practices in RTW interventions [11, 64, 65]. Watanabe [66] found that greater gains in cognitive functions can be achieved through a multidisciplinary approach to rehabilitation. Rattok and Ross [67] stated that for effective results, cognitive deficits should be treated within a comprehensive neurorehabilitation model involving multiple modalities. Turkstra and Kennedy [68] highlighted the collaboration between OTs and speech language pathologists (SLPs) to effectively deliver cognitive rehabilitation.

\subsection{Implications for practice}

- Cognitive assessment and rehabilitation deserves more attention across the continuum of TBI rehabilitation (acute, post-acute, and community-based).

- There is a need for a comprehensive RTW cognitive assessment tool specific to the TBI population.

- Return to work cognitive assessments should place more emphasis on executive functioning, attention, memory, information processing, and verbal skills, as these cognitive skills were found to increase the likelihood of successful RTW.

- With respect to RTW interventions, a multidisciplinary approach yields a better outcome. When facilitating RTW post TBI, successful job placements can be achieved when OTs, PTs, SLPs, psychologists, vocational counselors, social workers, and job coaches work together. 
- Compensatory cognitive strategies with supportive devices appear to be more effective than remedial strategies when facilitating RTW and community integration post TBI. Hence, therapists may consider using more of a compensatory model than a remedial model when treating patients with chronic cognitive impairments post TBI.

\subsection{Limitations}

First, articles included in this review were critically appraised by a single author. This may have resulted in selective perception/interpretation bias. Authors recognize this as a methodological flaw. However, to increase the validity of the findings, the second and third authors reviewed the critically appraised summaries after reading the originally published abstracts. Second, this review included only published literature; inclusion of gray literature may have reduced publication bias. Third, a majority of the studies included in this review recruited samples through convenience sampling, which increases sampling bias and limits the generalizability of the findings. However, the authors noted that, in some studies, to minimize the selection bias, participants were randomly assigned to the experiment and control groups after being recruited through selective sampling. Fourth, several intervention studies included in this review had low sample size, which limits the generalization of their findings and therefore, this review's findings. Further, in some intervention studies, though the primary focus was on cognitive rehabilitation, there appears to be an overlap of other related interventions, which means that the outcomes cannot strictly be attributed to cognitive interventions.

\section{Conclusion}

This systematic review asserts that cognition predicts and facilitates RTW post TBI. It also supports the notion that unemployment issues among TBI survivors can be mitigated through multidisciplinary care coordination with an increased focus on cognitive assessment and rehabilitation. Longitudinal studies are recommended to gain an in-depth understanding about the relationship between cognition and RTW post TBI, effects of cognitive rehabilitation on job sustainability, and factors influencing the long term delivery of cognitive rehabilitation, such as reimbursement and lost follow-up. Advocacy for cognitive rehabilitation to support RTW after TBI is recommended.

\section{Acknowledgments}

We acknowledge the assistance rendered by Shaun Conway and Pui-man Mak in the preparation and revision of the manuscript.

\section{Conflict of interest}

None to report.

\section{References}

[1] Centre for Disease Control and Prevention. Rates of TBIrelated emergency department visits, hospitalizations, and deaths - United States, 2001-2010. United States: CDC; 2016 [updated 2016 Jan 22; cited 201 Jun 4]. Available from: http://www.cdc.gov/traumaticbraininjury/data/rates.html.

[2] Texas Brain Injury Alliance. Brain injury statistics. Texas: The Alliance; 2016 [cited 2016 Jun 4]. Available from: http://www.texasbia.org/brain-injury-statistics.

[3] Vestling M, Tufvesson B, Iwarsson S. Indicators for return to work after stroke and the importance of work for subjective well-being and life satisfaction. J Rehab Med 2003;35:127.

[4] Harder H. Early intervention in disability management: Factors that influence successful return to work. Int $\mathrm{J}$ of Dis Comm and Rehab 2003;2. Available from: http:// www.ijdcr.ca/VOL02_02_CAN/articles/harder2.shtml

[5] Mackenzie A, Alfred D, Fountain R, Combs D. Quality of life and adaptation for traumatic brain injury survivors: Assessment of the disability centrality model. J Rehab 2015;81(3):9.

[6] Pierce C, Hanks R. Life satisfaction after traumatic brain injury and the World Health Organization model of disability. Am J of Phys Med Rehab 2006;85:889.

[7] Department of Defense \& Department of Veterans Affairs. Clinical practice guidelines for management of concussion/mild traumatic brain injury. Washington: DoD DVA; 2009.

[8] McNamee S, Walker W, Cifu DX, Wehman PH. Minimizing the effect of TBI-related physical sequelae on vocational return. J of Rehab Res and Dev 2009;46(6):893.

[9] Mahar C, Fraser K. Barriers to successful community reintegration following acquired brain injury. Int $\mathrm{J}$ of Dis Management Res 2011;6(1):49.

[10] Boake C, Millis SR, High WM, Delmonico RL, Kreutzer JS, Rosenthal M, et al. Using early neuropsychologic testing to predict long-term productivity outcome from traumatic brain injury. Arch Phys Med Rehab 2001;82(6):761.

[11] Boeing M, Barton B, Zinsmeister P, Brouwers L, Trudel TM, Elias E, et al. Lifelong living after TBI. The Exceptional Parent 2010;40(10):32.

[12] Coetzer BR, Hayes NM, Du Toit PL. Long-term employment outcomes in a rural area following traumatic brain injury. Aus J Rural Health 2002;10(4):229. 
[13] van Velzen JM, van Bennekom, CAM, Edelaar MJA, Sluiter JK, Frings-Dresen M. How many people return to work after acquired brain injury?: A systematic review. Brain Inj 2009;23(6):473.

[14] Doctor JN, Castro J, Temkin NR, Fraser RT, Machamer JE, Dikmen SS. Workers' risk of unemployment after traumatic brain injury: A normed comparison. J Int Neuropsy Society 2005;11(6):747.

[15] Draper K, Ponsford J. Cognitive functioning ten years following traumatic brain injury and rehabilitation. Neuropsy 2008;22(5):618.

[16] Foy CML. Educational Attainment and Ability in Young Adults Following Acquired Brain Injury. J Occ Rehab 2015;25(2):394.

[17] Fong KNK, Howie DR. Effects of an explicit problemsolving skills training program using a metacomponential approach for outpatients with acquired brain injury. Am J Occ Ther 2009;3(5):525.

[18] Madigan NK, DeLuca J, Diamond BJ, Tramontano G, Averill A. Speed of information processing in traumatic brain injury: Modality-specific factors. J Head Trauma Rehab 2000;15(3):943.

[19] Cooper DB, Bunner AE, Kennedy JE, Balldin V, Tate DF, Eapen $\mathrm{BC}$, et al. Treatment of persistent post-concussive symptoms after mild traumatic brain injury: A systematic review of cognitive rehabilitation and behavioral health interventions in military service members and veterans. Brain Imaging and Beh 2015;9(3):403.

[20] Hammond FM, Hart T, Bushnik T, Corrigan JD, Sasser $\mathrm{H}$. Change and predictors of change in communication, cognition, and social function between 1 and 5 Years after traumatic brain injury. J Head Trauma Rehab 2004;19(4):314.

[21] Keyser-Marcus L, Bricout JC, Wehman P, Campbell LR, Cifu DX, Englander J, et al. Acute predictors of return to employment after traumatic brain injury: A longitudinal follow-up. Arch Phys Med Rehab 2002;83(5):635.

[22] Schonbrun SL, Kampfe CM. Variables relating to employment outcome in individuals with traumatic brain injury. J of Appl Rehab Coun 2004;35(3):14.

[23] Drake AI, Gray N, Yoder S, Pramuka M, Llewellyn M. Factors predicting return to work following mild traumatic brain injury: A discriminant analysis. J Head Trauma Rehab 2000;15(5):1103.

[24] Leung KL, Man DWK. Prediction of vocational outcome of people with brain injury after rehabilitation: A discriminant analysis. Work 2005;25(4):333.

[25] Stergiou-kita M, Dawson DR, Rappolt SG. An Integrated Review of the Processes and Factors Relevant to Vocational Evaluation Following Traumatic Brain Injury. J Occ Rehab 2011;21(3):374.

[26] Benedictus MR, Spikman JM, Van DN. Cognitive and behavioral impairment in traumatic brain injury related to outcome and return to work. Arch Phys Med Rehab 2010;91(9):1436.

[27] Stergiou-Kita M, Mansfield E, Sokoloff S, Colantonio A. Gender influences on return to work after mild traumatic brain injury. Arch Phys Med Rehab 2016;97(2):S40.

[28] Satoko Y. Participant characteristics and support services that influence successful employment outcomes of social security beneficiaries with traumatic brain injury. Ann Arbor: Virginia Commonwealth University; 2003.

[29] LoBiondo-Wood G. Systematic reviews and clinical practice guidelines. In: LoBiondo-Wood G, Haber J, editors. Nursing research: Methods and critical appraisal for evi- dence based practice. 8th ed. St. Louis: Elsevier; 2014. p. 218.

[30] Escorpizo R, Reneman MF, Ekholm J, Fritz J, Krupa T, Marnetoft $\mathrm{S}$, et al. A conceptual definition of vocational rehabilitation based on the ICF: Building a shared global model. J Occ Rehab 2011;21(2):126.

[31] Brain Injury Association of America. What is a difference between an acquired brain injury and a traumatic brain injury? Virginia: BIAA; 2015 [cited 2016 Jun 5]. Available from: http://www.biausa.org/FAQRetrieve.aspx?ID=43913 $\& A=$ SearchResult $\&$ SearchID=9758947\&ObjectID=43913 \&ObjectType $=9$.

[32] American Occupational Therapy Association. Guidelines for systematic reviews. Maryland: AOTA; 2014 [updated 2015 Dec; cited 2016 Jun 5]. Available from: http://ajot.submit2aota.org/journals/ajot/forms/systematic_ reviews.pdf

[33] Willemse-van Son AHP, Ribbers GM, Verhagen AP, Stam HJ. Prognostic factors of long-term functioning and productivity after traumatic brain injury: A systematic review of prospective cohort studies. Clinical Rehab 2007;21(11):1024.

[34] Ownsworth T, McKenna K. Investigation of factors related to employment outcome following traumatic brain injury: A critical review and conceptual model. Dis Rehab 2004;26(13):765.

[35] Watt N, Penn C. Predictors and indicators of return to work following traumatic brain injury in South Africa: Findings from a preliminary experimental database. S Afr J Psychol 2000;30(2):27.

[36] Kreutzer JS, Marwitz JH, Walker W, Sander A, Sherer M, Bogner J, et al. Moderating factors in return to work and job stability after traumatic brain injury. J Head Trauma Rehab 2003;18(2): 128 .

[37] Scherer MR, Weightman MM, Radomski MV, Davidson LF, McCulloch KL. Returning service members to duty following mild traumatic brain injury: Exploring the use of dual-task and multitask assessment methods. Phys Ther 2013;93(9):1254

[38] Chappell I, Higham J, McLean AM. An occupational therapy work skills assessment for individuals with head injury. Can J of Occ Ther 2003;70(3):163.

[39] Stergiou-Kita M, Rappolt S, Kirsh B, Shaw L. Evaluating work readiness following acquired brain injury: Building a shared understanding. Can J of Occ Ther 2009;76(4):276.

[40] Vandiver VL, Johnson J, Christofero-Snider C. Supporting employment for adults with acquired brain injury: A conceptual model. J Head Trauma Rehab 2003;18(5):457.

[41] Bootes K, Chapparo C. Difficulties with multitasking on return to work after TBI: A critical case study. Work 2010;36(2):207.

[42] Shames J, Treger I, Ring H, Giaquinto S. Return to work following traumatic brain injury: Trends and challenges. Disability and Rehab 2007;29(17):1387.

[43] Wehman P, Gentry, Tony, West M, Arango-Lasprilla J. Community integration: Current issues in cognitive and vocational rehabilitation for individuals with ABI. J of Rehab Res and Dev 2009;46(6):909.

[44] Trudel TM, Nidiffer FD, Barth JT. Community-integrated brain injury rehabilitation: Treatment models and challenges for civilian, military, and veteran populations. J Rehab Res and Dev 2007;44(7):1007.

[45] Twamley EW, Jak AJ, Delis DC, Bondi MW, Lohr JB. Cognitive symptom management and rehabilitation therapy (CogSMART) for veterans with traumatic brain injury: 
Pilot randomized controlled trial. J Rehab Res and Dev 2014;51(1):59.

[46] Man DWK, Poon WS, Lam C. The effectiveness of artificial intelligent 3-D virtual reality vocational problem-solving training in enhancing employment opportunities for people with traumatic brain injury. Brain Inj 2013;27(9):1016.

[47] Radford K, Phillips J, Drummond A, Sach T, Walker M, Tyerman A, et al. Return to work after traumatic brain injury: Cohort comparison and economic evaluation. Brain Inj 2013;27(5):507.

[48] Cicerone KD, Mott T, Azulay J, Friel JC. Community integration and satisfaction with functioning after intensive cognitive rehabilitation for traumatic brain injury. Arch Phys Med Rehab 2004;85(6):943.

[49] Klonoff PS, Talley MC, Dawson LK, Myles SM, Watt LM, Gehrels J, et al. The relationship of cognitive retraining to neurological patients' work and school status. Brain Inj 2007;21(11):1097.

[50] Mateer CA, Sira CS. Cognitive and emotional consequences of TBI: Intervention strategies for vocational rehabilitation. NeuroRehab 2006;21(4):315.

[51] Radomski MV, Davidson L, Voydetich D, Erickson MW. Occupational Therapy for service members with mild traumatic brain injury. Am J Occ Ther 2009;63(5):646.

[52] Walker WC, Marwitz JH, Kreutzer JS, Hart T, Novack TA. Occupational categories and return to work after traumatic brain injury: A multicenter study. Arch Phys Med Rehab 2006;87(12):1576.

[53] Matheson LN, Dodson MB, Wolf TJ. Executive dysfunction and work: Tying it all together. AOTA Work and Industry SIS Quarterly 2011;25(1):1.

[54] Yasuda S, Wehman P, Targett P, Cifu D, West M: Return to work for persons with traumatic brain injury. Am J Phys Med Rehab 2001;80:852.

[55] O'Connell MJ. Prediction of return to work following traumatic brain injury: Intellectual, memory, and demographic variables. Rehab Psy 2000;45(2):212.

[56] Marsh N, Smith M. Post-concussion syndrome and the coping hypothesis. Brain Inj 1995;9(6):553.

[57] Ruff RM, Jurica P. In search of unified definition for mild traumatic brain injury. Brain Inj 1999;13:943.
[58] Stergiou-kita M, Dawson D, Rappolt S. Inter-Professional clinical practice guideline for vocational evaluation following traumatic brain injury: A systematic and evidence-based approach. J Occ Rehab 2012;22(2):166.

[59] Wasiak R, Young AE, Roessler RT, McPherson KM, van Poppel MNM, et al. Measuring return to work. J Occ Rehab 2007;17(4):766.

[60] Hoofien D, Vakil E, Gilboa A, Donovick PJ, Barak O. Comparison of the predictive power of socio-economic variables, severity of injury and age on long-term outcome of traumatic brain injury: Sample-specific variables versus factors as predictors. Brain Inj 2002;16(1):9.

[61] Bayley MT, Tate R, Douglas JM, Turkstra LS, Ponsford J, Stergiou-Kita M, Kua A, Bragge P, INCOG expert panel. INCOG guidelines for cognitive rehabilitation following traumatic brain injury: Methods and overview. J Head Trauma Rehab 2014;29(4):290.

[62] Cicerone KD, Langenbahn DM, Braden C, Malec JF, Kalmar K, Fraas M, Azulay J, et al. Evidence-based cognitive rehabilitation: Updated review of literature through 2003 through 2011. Arch of Phys Med and Rehab 2000;92(4):519.

[63] Cernich AN, Kurtz SM, Mordecai KL, Ryan PB. Cognitive rehabilitation in traumatic brain injury. Current Treatment Options in Neurology 2010;12(5):412.

[64] Conradie LJ, Stewart A. Return to work after head injury. Occ Health 2008;60(4):43.

[65] Bogdanova Y, Verfaellie M. Cognitive sequelae of blastinduced traumatic brain injury: Recovery and rehabilitation. Neuropsy Review 2012;22(1):4.

[66] Watanabe S. Vocational rehabilitation for clients with cognitive and behavioral disorders associated with traumatic brain injury. Work 2013;45(2):273.

[67] Rattok J, Ross BP. A practical approach to cognitive rehabilitation. Neurorehab 1992;2(3):31.

[68] Turkstra LS, Kennedy MRT. Traumatic brain injury and cognitive rehabilitation. Am Speech and Hearing Assn Leader 2008;13(9): 10 . 